\title{
Comparison of effectiveness and safety of imipenem/clavulanate- versus meropenem/clavulanate-containing regimens in the treatment of MDR- and XDR-TB
}

Simon Tiberi ${ }^{1,30}$, Giovanni Sotgiu ${ }^{2,30}$, Lia D’Ambrosio ${ }^{3,4,30}$, Rosella Centis ${ }^{3,30}$, Marcos Abdo Arbex ${ }^{5,6}$, Edith Alarcon Arrascue ${ }^{7,8}$, Jan Willem Alffenaar ${ }^{9}$, Jose A. Caminero ${ }^{8,10}$, Mina Gaga ${ }^{11}$, Gina Gualano ${ }^{12}$, Alena Skrahina ${ }^{13}$, Ivan Solovic ${ }^{14}$, Giorgia Sulis ${ }^{15}$, Marina Tadolini ${ }^{16}$, Valentina Alarcon Guizado ${ }^{17}$, Saverio De Lorenzo ${ }^{18}$, Aurora Jazmín Roby Arias ${ }^{19}$, Anna Scardigli ${ }^{8}$, Onno W. Akkerman ${ }^{20}$, Alena Aleksa ${ }^{21}$, Janina Artsukevich ${ }^{21}$, Vera Auchynka ${ }^{13}$, Eduardo Henrique Bonini ${ }^{5,6}$, Félix Antonio Chong Marín ${ }^{19}$, Lorena Collahuazo López ${ }^{19}$, Gerard de Vries ${ }^{22}$, Simone Dóre ${ }^{2}$, Heinke Kunst ${ }^{23}$, Alberto Matteelli ${ }^{15}$, Charalampos Moschos ${ }^{11}$, Fabrizio Palmieri ${ }^{12}$, Apostolos Papavasileiou ${ }^{11}$, Marie-Christine Payen ${ }^{24}$, Andrea Piana ${ }^{2}$, Antonio Spanevello ${ }^{25,26}$, Dante Vargas Vasquez ${ }^{27}$, Pietro Viggiani ${ }^{18}$, Veronica White ${ }^{28}$, Alimuddin Zumla ${ }^{29}$ and Giovanni Battista Migliori ${ }^{3}$

ABSTRACT No large study to date has ever evaluated the effectiveness, safety and tolerability of imipenem/clavulanate versus meropenem/clavulanate to treat multidrug- and extensively drug-resistant tuberculosis (MDR- and XDR-TB). The aim of this observational study was to compare the therapeutic contribution of imipenem/clavulanate versus meropenem/clavulanate added to background regimens to treat MDR- and XDR-TB cases.

84 patients treated with imipenem/clavulanate-containing regimens showed a similar median number of antibiotic resistances ( 8 versus 8 ) but more fluoroquinolone resistance $(79.0 \%$ versus $48.9 \%, \mathrm{p}<0.0001)$ and higher XDR-TB prevalence (67.9\% versus $49.0 \%, \mathrm{p}=0.01$ ) in comparison with 96 patients exposed to meropenem/clavulanate-containing regimens. Patients were treated with imipenem/clavulanate- and meropenem/clavulanate-containing regimens for a median (interquartile range) of 187 (60-428) versus 85 (49-156) days, respectively.

Statistically significant differences were observed on sputum smear and culture conversion rates $(79.7 \%$ versus $94.8 \%, \mathrm{p}=0.02$ and $71.9 \%$ versus $94.8 \%, \mathrm{p}<0.0001$, respectively) and on success rates (59.7\% versus $77.5 \%, \mathrm{p}=0.03$ ). Adverse events to imipenem/clavulanate and meropenem/clavulanate were reported in $5.4 \%$ and $6.5 \%$ of cases only.

Our study suggests that meropenem/clavulanate is more effective than imipenem/clavulanate in treating MDR/XDR-TB patients.

@ERSpublications

Meropenem/clavulanate is safe and more effective than imipenem/clavulanate in treating MDR and XDR-TB patients http://ow.ly/Z4S2o 
Affiliations: 'Division of Infection, Barts Healthcare NHS Trust, London, UK. ${ }^{2}$ Clinical Epidemiology and Medical Statistics Unit, Dept of Biomedical Sciences, University of Sassari - Research, Medical Education and Professional Development Unit, AOU Sassari, Sassari, Italy. ${ }^{3}$ WHO Collaborating Centre for TB and Lung Diseases, Fondazione S. Maugeri, Tradate, Italy. ${ }^{4}$ Public Health Consulting Group, Lugano, Switzerland. ${ }^{5}$ University Center of Araraquara, Sao Paulo, Brazil. ${ }^{6}$ Hospital Nestor Goulart Reis, Sao Paulo State Secretary of Health, Sao Paulo, Brazil. ${ }^{7}$ International Union against Tuberculosis and Lung Disease (The Union), Lima, Peru. ${ }^{8}$ MDR-TB Unit, Tuberculosis Division, International Union against Tuberculosis and Lung Disease (The Union), Paris, France. ${ }^{9}$ University of Groningen, University Medical Center Groningen, Dept of Clinical Pharmacy and Pharmacology, Groningen, The Netherlands. ${ }^{10}$ Pneumology Dept, Hospital General de Gran Canaria "Dr. Negrin", Las Palmas de Gran Canaria, Spain. ${ }^{11}$ MDR-TB Unit, Athens Chest Hospital Sotira, National Referral Centre for Mycobacteria, Athens, Greece. ${ }^{12}$ Respiratory Infectious Diseases Unit, National Institute for Infectious Diseases “L. Spallanzani”, Rome, Italy. ${ }^{13}$ Republican Research and Practical Centre for Pulmonology and Tuberculosis, Minsk, Belarus. ${ }^{14}$ National Institute for TB, Lung Diseases and Thoracic Surgery, Vysne Hagy, Catholic University Ruzomberok, Ruzomberok, Slovakia. ${ }^{15}$ University Dept of Infectious and Tropical Diseases, WHO Collaborating Centre for TB/HIV Co-infection and for TB Elimination - University of Brescia and Brescia Spedali Civili General Hospital, Brescia, Italy. ${ }^{16}$ Unit of Infectious Diseases, Dept of Medical and Surgical Sciences, Alma Mater Studiorum University of Bologna, Bologna, Italy. ${ }^{17}$ National Tuberculosis Control Programme, Ministry of Health, Lima, Peru. ${ }^{18}$ AOVV E. Morelli Hospital, Reference Hospital for MDR and HIV-TB, Sondalo, Italy. ${ }^{19}$ National Tuberculosis Control Programme, Pulmonology Hospital Alfredo J. Valenzuela, Guayaquil, Ecuador. ${ }^{20}$ University of Groningen, University Medical Center Groningen, Dept of Pulmonary Diseases and Tuberculosis; TB Center Beatrixoord, Groningen, The Netherlands. ${ }^{21}$ Educational Institution "Grodno State Medical University", Grodno, Belarus. ${ }^{22}$ KNCV Tuberculosis Foundation, The Hague, The Netherlands. ${ }^{23}$ Dept of Respiratory Medicine, Queen Mary University, London, UK. ${ }^{2}$ Division of Infectious Diseases, CHU Saint-Pierre, Université Libre de Bruxelles (ULB), Brussels, Belgium. ${ }^{25}$ Pneumology Unit, Fondazione Maugeri, IRCCS, Tradate, Italy. ${ }^{26}$ Dept of Clinical and Experimental Medicine, University of Insubria, Varese, Italy. ${ }^{27}$ National Hospital Hipólito Unanue, Public Health Institute, Ministry of Health, Lima, Peru. ${ }^{28}$ Dept of Respiratory Medicine, Barts Healthcare NHS Trust, London, UK. ${ }^{29}$ Division of Infection and Immunity, University College London and NIHR Biomedical Research Centre, UCL Hospitals NHS Foundation Trust, London, UK. ${ }^{30}$ These authors contributed equally to this work.

Correspondence: Giovanni Battista Migliori, World Health Organization Collaborating Centre for Tuberculosis and Lung Diseases, Fondazione S. Maugeri, Care and Research Institute, Via Roncaccio 16, 21049, Tradate, Italy. E-mail: giovannibattista.miglioriafsm.it

\section{Introduction}

Over 480000 new multidrug-resistant tuberculosis (MDR-TB) cases and 190000 deaths were estimated to occur in 2014 by the World Health Organization (WHO), with only one MDR-TB case out of four being currently diagnosed and treated [1]. Half of the 123000 cases of MDR-TB notified to the WHO in 2014 occurred in India, the Russian Federation and South Africa. Globally, 3.3\% of new cases and 20\% of previously treated cases of TB harbour MDR-TB strains of Mycobacterium tuberculosis. While overall 9.7\% of the MDR-TB strains met the criteria defining extensively drug-resistant (XDR)-TB (e.g. resistance to at least one fluoroquinolone and a second-line injectable drug), this proportion was much higher in some of the former Soviet Union countries (Belarus 29\%, Latvia 15\% and Georgia 15\%) [1, 2].

Recent evidence shows that treating MDR- and XDR-TB patients with the drugs available today is long, expensive, complicated and associated with frequent adverse events [3-8].

Presently a stepwise approach in the use of second-line anti-TB drugs (which are classified into five groups) is recommended by WHO [7, 9].

The main difficulty faced by clinicians treating MDR- and XDR-TB cases is to identify at least four active drugs which are necessary to design an effective multidrug regimen as per the WHO guidelines $[3,4,7$, 10, 11]. New drugs, such as delamanid, [12-14] and bedaquiline [15-20], and a few repurposed drugs (presently included in WHO Group 5, i.e. drugs with unknown/limited evidence on efficacy and/or tolerability) are presently attracting scientific interest [7, 21], among them linezolid [10, 22-28] and carbapenems [29-34]. The carbapenem group of drugs, which includes meropenem, imipenem and ertapenem, are currently used to treat MDR- and XDR-TB cases, although the evidence available on their efficacy, safety and tolerability is extremely limited [29-34].

The effectiveness of $\beta$-lactam antibiotics is unfortunately limited, as M. tuberculosis is protected through its potent $\beta$-lactamase, encoded by the blaC gene $[35,36]$. Recent studies indicate that clavulanate (a $\beta$-lactamase inhibitor) can inhibit the activity of blaC-coded products in vitro [36]. Meropenem, offering a limited substrate to hydrolysis, has shown high bactericidal in vitro activity in association to clavulanate against $M$. tuberculosis (susceptible, MDR- and XDR-TB strains) as well as the in vivo ability to sterilise cultures within 2 weeks $[37,38]$. Moreover, a study suggested synergy with amoxicillin and meropenem [39]. This has been confirmed in a murine model [40]. Its effectiveness, initially suggested in two case reports of 10 cases or less [30,41], was then confirmed by two case-control studies. The first, with a limited sample size ( 37 patients at a single centre), suggested the drug was effective (offering up to 
20-30\% sterilising power) and well tolerated [29]. The second, more recent study, was conducted by the International Carbapenems Study Group (ICSG, formerly the European Carbapenems Study Group) in 17 centres and six countries in Europe and Latin America with over 90 cases. It confirmed that meropenem/ clavulanate is safe and that the activity of the drug combination is promising, as nondifferent (noninferior) sputum smear conversion, culture conversion and treatment success rates between cases and controls have been identified despite controls having a much less severe resistance pattern than meropenem/ clavulanate-treated cases [42].

Evidence on imipenem/clavulanate is even more limited, consisting of a series of 10 patients and one case report $[33,34]$.

Recently, the TB reference centres belonging to the ICSG [31,42] conducted a large observational study evaluating the therapeutic contribution of imipenem/clavulanate added to a background regimen (as per the WHO guidelines) when treating MDR- and XDR-TB cases.

The aim of the present study was to compare the effectiveness, safety and tolerability profile of meropenem/clavulanate and imipenem/clavulanate in a large observational cohort of MDR-TB patients.

\section{Material and methods}

The methodological characteristics were previously described [42]. MDR-TB reference centres located in European and Southern American countries recruited culture-confirmed MDR-TB patients aged $\geqslant 15$ years. An MDR-TB case was defined as an individual with TB caused by M. tuberculosis strains phenotypically resistant to at least isoniazid and rifampicin. Patients were consecutively selected on the basis of their exposure to meropenem/clavulanate and imipenem/clavulanate during their intensive and/or continuation phase.

An individualised TB regimen was administered following the results of the drug-susceptibility test (DST) carried out by externally quality-assured laboratories [11]. The attending physician prescribed anti-TB drugs without any compelling criteria of experimental protocols and, consequently, blinding or randomised methods were not followed. Meropenem/clavulanate was administered at a daily dosage of meropenem $1 \mathrm{~g}$ three times daily intravenously plus amoxicillin/clavulanic acid $1.2 \mathrm{~g}$ three times daily. The only exception was represented by the Belgium centre where meropenem was prescribed at a daily dosage of $2 \mathrm{~g}$ three times daily $(17 / 180,9.4 \%)$. Imipenem/clavulanate was administered at a dose of $500 \mathrm{mg}$ imipenem four times daily plus amoxicillin/clavulanic acid as above.

Standardised ad hoc E-forms were prepared to collect epidemiological (i.e. duration of hospital stay, age, place of birth, sex, residence, immigration from a TB high-burden country), clinical (i.e. HIV status, administration of HIV drugs, previous TB diagnosis and treatment, previous treatment outcomes, radiological findings, TB therapy and related adverse events, duration of exposure to meropenem/ clavulanate and imipenem/clavulanate, surgery, sputum smear and culture positivity at the treatment baseline, at 30, 60 and 90 days, time to sputum smear and culture conversion, WHO treatment outcomes) and microbiological (i.e. DST results) information from official medical files.

Qualitative and quantitative variables were summarised with percentages and mean \pm SD or median (interquartile range (IQR)) depending on their normality. The Chi-squared or Fisher's exact and the Mann-Whitney test were adopted to compare qualitative and quantitative variables, respectively. Differences in terms of sputum smear and culture conversions between imipenem/clavulanate and meropenem/clavulanate groups were assessed with a survival analysis and a log-rank test. A p-value $<0.05$ (two-tailed) was considered statistically significant. Statistical computations were performed with Stata 13.0 (StataCorp, College Station, TX, USA).

Ethical approval for the collection and analysis of anonymous and retrospective data and for the compassionate use of the drugs was obtained by the institutional review boards of the participating institutions as per legislation in force in the different ICSG countries and at the coordinating centre.

\section{Results}

180 MDR-TB patients were selected for the analysis: 96 (53.3\%) exposed to meropenem/clavulanate and $84(46.7 \%)$ exposed to imipenem/clavulanate (table 1).

The prevalent sex of the cohort was male $(105,58.3 \%)$ and the median (IQR) age was 35 (26-45) years (table 1).

Migrants coming from high TB prevalence countries accounted for 100 (55.6\%) patients, whereas only a low proportion (5.8\%) were HIV-infected. A higher proportion of migrants $(76.0 \%$ versus $32.1 \%, \mathrm{p}<0.0001)$ and of patients born in Europe $(75.0 \%$ versus $45.2 \%, \mathrm{p}<0.0001)$ was observed in the meropenem/clavulanate-treated group (table 1). 
TABLE 1 Demographic, epidemiological and clinical characteristics of multidrug- and extensively drug-resistant tuberculosis (MDR- and XDR-TB) patients treated with meropenem/clavulanate (MC)-versus imipenem/clavulanate (IC)-containing regimens

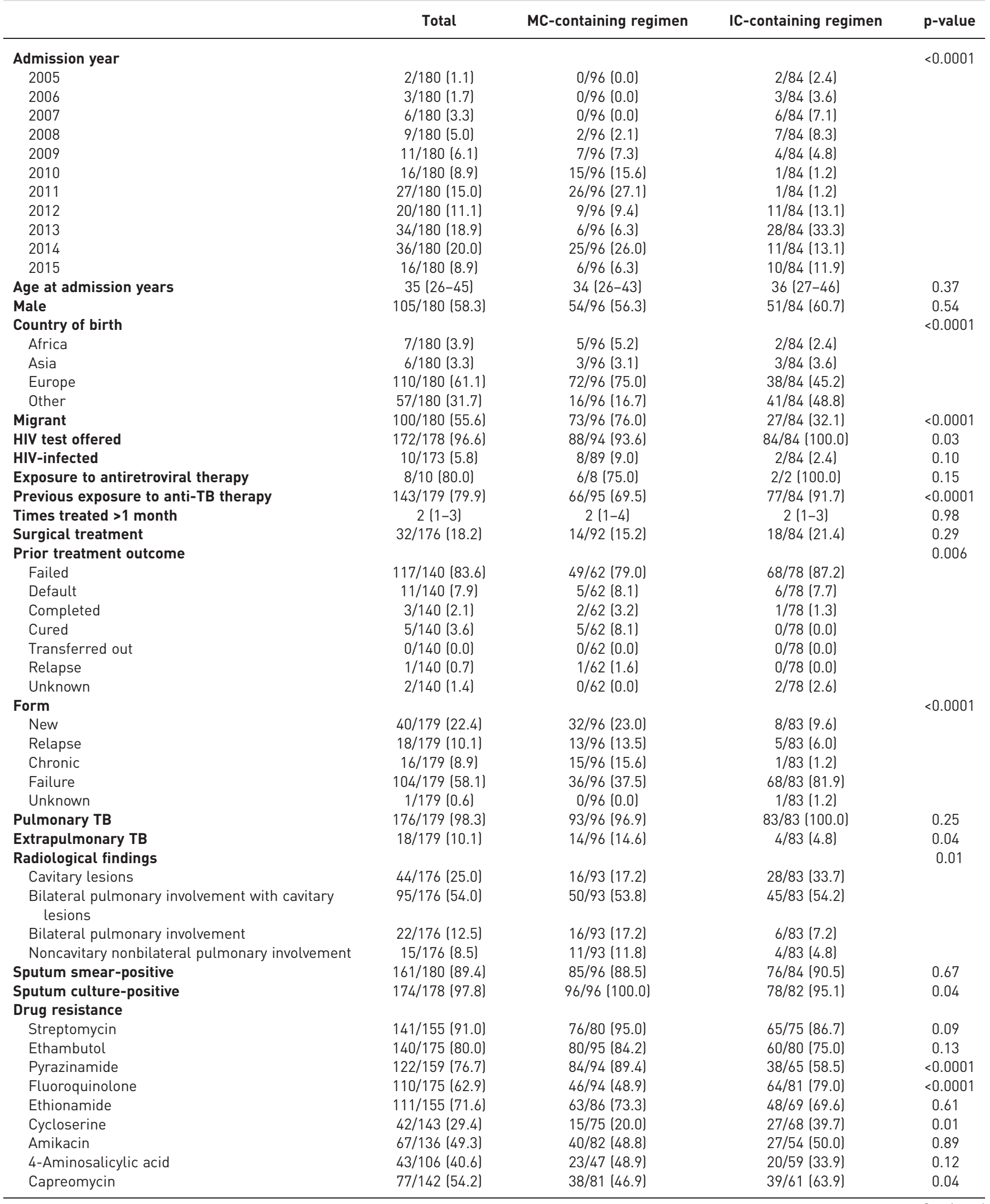


TABLE 1 Continued

\begin{tabular}{|c|c|c|c|c|}
\hline & Total & MC-containing regimen & IC-containing regimen & p-value \\
\hline Kanamicin & $84 / 137$ (61.3) & $37 / 75(49.3)$ & 47/62 (75.8) & 0.002 \\
\hline Linezolid & $5 / 83(6.0)$ & 4/75 (5.3) & $1 / 8$ (12.5) & 0.41 \\
\hline Other & $10 / 18(55.6)$ & $6 / 11(54.6)$ & 4/7 (57.1) & 1.00 \\
\hline Antibiotic resistances & $8(6-9)$ & $8(6-9)$ & $8(7-8)$ & 0.34 \\
\hline Length of hospital stay days & $129(68-206)$ & $101(61-172)$ & $164(110-247)$ & 0.0001 \\
\hline Moxifloxacin & 145/179 (81.0) & $77 / 95(81.1)$ & $68 / 84(81.0)$ & 0.99 \\
\hline Levofloxacin & $20 / 180(11.1)$ & $9 / 96(9.4)$ & $11 / 84(13.1)$ & 0.43 \\
\hline Linezolid & $97 / 166(58.4)$ & $50 / 90(55.6)$ & 47/76 (61.8) & 0.41 \\
\hline Delamanid & $1 / 175(0.6)$ & $1 / 95(1.1)$ & $0 / 80(0.0)$ & 1.00 \\
\hline Bedaquiline & $16 / 176(9.1)$ & $9 / 95$ (9.5) & $7 / 81(8.6)$ & 0.85 \\
\hline
\end{tabular}

Data are presented as $\mathrm{n} / \mathrm{N}(\%)$ or median (interquartile range), unless otherwise stated. Denominators ( $\mathrm{N}$ ) were different among the selected variables because of missing data.

Pulmonary TB was diagnosed in 176 out of 179 (98.3\%) cases. The proportion of sputum smear- and culture-positive cases was $89.4 \%$ and $97.8 \%$, respectively (table 2 ).

More than half were affected by XDR-TB (57.8\%; 67.9\% and $49.0 \%$ in the imipenem/clavulanate and meropenem/clavulanate group, respectively, $\mathrm{p}=0.01$ ), but the median number of drug resistances was similar ( 8 versus 8 in the meropenem/clavulanate and imipenem/clavulanate group, respectively, $\mathrm{p}=0.34$ ) (table 2).

Over three-quarters of cases were previously treated for drug-susceptible or -resistant TB; however, patients exposed to imipenem/clavulanate-containing regimens had been treated more frequently for a previous TB diagnosis $(91.7 \%$ versus $69.5 \%, \mathrm{p}<0.0001)$. However, the median (IQR) number of previous anti-TB treatments was similar in the meropenem/clavulanate- and imipenem/clavulanate-treated groups (2 (1-4) versus $2(1-3))$ (table 1$)$.

The prevalence of resistance to any fluoroquinolone was significantly higher among the imipenem/ clavulanate-treated cases $(79.0 \%$ versus $48.9 \%, \mathrm{p}<0.0001)$; moreover, a higher prevalence of resistance to capreomycin $(63.9 \%$ versus $46.9 \%, \mathrm{p}=0.04)$ and kanamycin $(75.8 \%$ versus $49.3 \%, \mathrm{p}<0.002)$ was observed in comparison with the meropenem/clavulanate-treated group. The prevalence of resistance to amikacin was similar in both groups (50.0\% versus $48.8 \%$, respectively) (table 1 ).

Patients were treated with meropenem/clavulanate- and imipenem/clavulanate-containing regimens for a median (IQR) of 85 (49-156) and 187 (60-428) days (table 3). Adjuvant surgical therapy was performed in $18.2 \%$ of the cases.

Sputum smear and culture conversion rates were significantly higher in those exposed to meropenem/ clavulanate ( $94.8 \%$ and $94.8 \%$, respectively) than in those exposed to imipenem/clavulanate $(79.7 \%$ and $71.9 \%, p=0.02$ and $p<0.0001$, respectively). Similar proportional differences for the culture conversion were achieved when subgroup analyses were performed according to MDR/XDR-TB status and the median number of drug resistances (i.e. $\geqslant 8$ drug resistances) (table 2).

The time to culture conversion was shorter in the meropenem/clavulanate- than in the imipenem/ clavulanate-treated group (44 (28-75) versus 60 (30-90) days, $\mathrm{p}=0.05)$ (table 2 and figure 1 ).

Overall, cases treated with meropenem/clavulanate achieved higher statistically significant success rates than those treated with imipenem/clavulanate (77.5\% versus $59.7 \%, \mathrm{p}=0.03$ ) (table 2).

Adverse events were reported only in six (6.5\%) meropenem/clavulanate- and three (5.4\%) imipenem/ clavulanate-treated patients $(\mathrm{p}=1.0)($ table 3$)$.

\section{Discussion}

The aim of the present study was to retrospectively compare the effectiveness, safety and tolerability profile of meropenem/clavulanate and imipenem/clavulanate in a large cohort of XDR- and MDR-TB patients.

The results of our study demonstrated that, overall, the meropenem/clavulanate-containing regimens achieved better results than imipenem/clavulanate-containing regimens. In particular, 1) culture conversion rates were statistically higher (both in the whole cohort and in the XDR/MDR-TB subgroups), 
TABLE 2 Treatment outcomes of multidrug- and extensively drug-resistant tuberculosis (MDR- and XDR-TB) patients treated with meropenem/clavulanate (MC)-versus imipenem/clavulanate (IC)-containing regimens

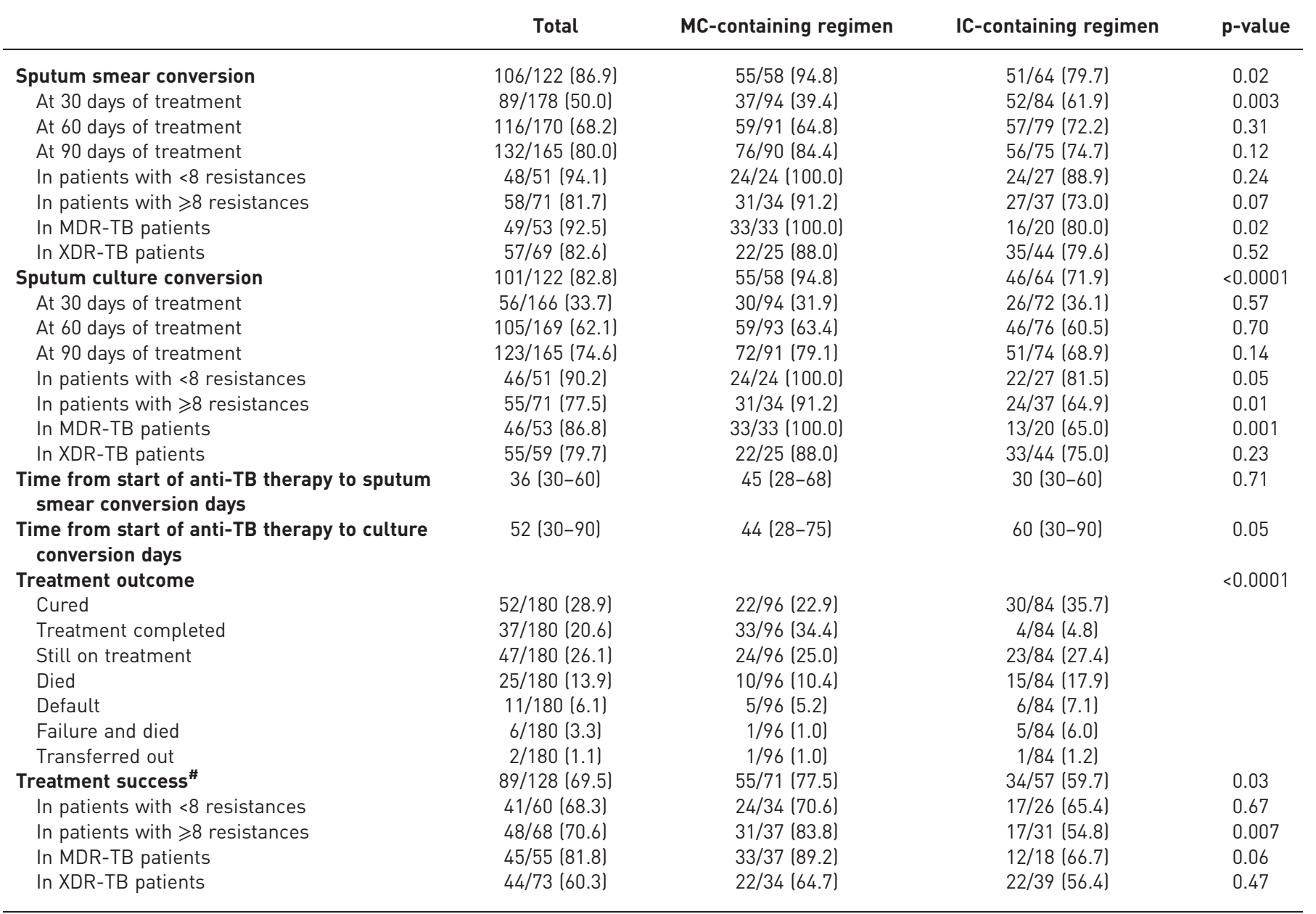

Data are presented as $\mathrm{n} / \mathrm{N}(\%)$ or median (interquartile range), unless otherwise stated. Denominators ( $\mathrm{N}$ ) were different among the selected variables because of missing data. ${ }^{\#}$ : denominator includes patients who were successfully treated, died, defaulted or failed.

2) the time to culture conversion was shorter and 3) the treatment success rates were significantly higher (both in the overall cohort and in the patients with $\geqslant 8$ resistances) in the meropenem/clavulanate- than in the imipenem/clavulanate-containing group.

Furthermore, the study demonstrated that both drugs were well tolerated and adverse events were rare (only $6.5 \%$ in the meropenem/clavulanate- and $5.4 \%$ in the imipenem/clavulanate-containing regimens).

This is, to the best of our knowledge, the first study in the scientific literature describing the efficacy, safety and tolerability of imipenem/clavulanate-containing regimens, and comparing the clinical performances of two of the core carbapenems (i.e. imipenem/clavulanate and meropenem/clavulanate) in a large cohort of XDR- and MDR-TB patients.

In a previous study by the same group [42] the performance of the meropenem/clavulanate-containing regimens was compared with a control group. The study results suggested that meropenem/clavulanate is active, as meropenem/clavulanate-containing regimens achieved nondifferent results than the controls in spite of a much more severe resistance profile.

Although it is always difficult to attribute the causality of the results observed to meropenem/clavulanate and imipenem/clavulanate, we can underline that imipenem/clavulanate-containing regimens achieved bacteriological and treatment success rates comparable to those of other cohorts of MDR-TB cases [3, 4], while meropenem/clavulanate-containing-regimens had promising results $(94.8 \%$ sputum smear and culture conversion, and $77.5 \%$ treatment success). In order to remove the "background noise" caused by the observational design, the study findings need to be confirmed by randomised controlled clinical trials. 
TABLE 3 Safety of meropenem/clavulanate (MC)-versus imipenem/clavulanate (IC)-containing regimens

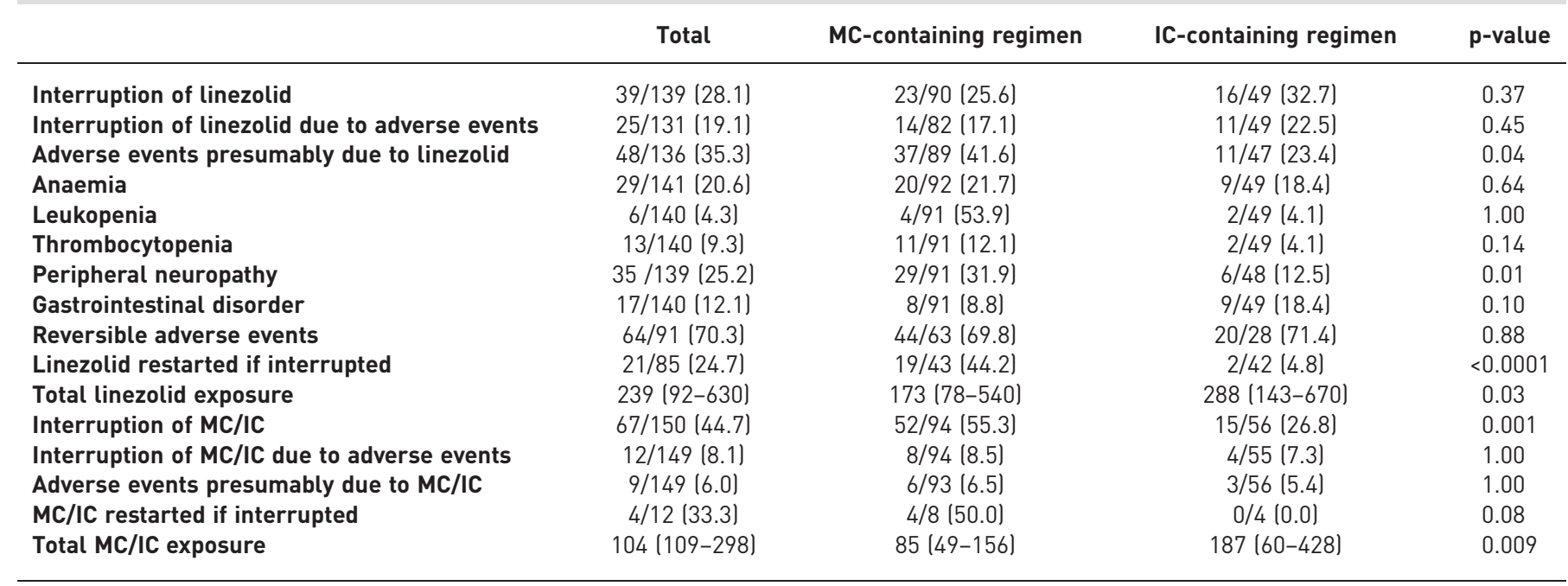

Data are presented as $\mathrm{n} / \mathrm{N}(\%)$ or median (interquartile range), unless otherwise stated. Denominators $(\mathrm{N})$ were different among the selected variables because of missing data.

Based on the molecular mechanism of action, imipenem is more active than meropenem [43]. However, this does not necessarily translate into better clinical results. The pharmacokinetic/pharmacodynamic index, as well as the free drug concentration exceeding the minimal inhibitory concentration, could have favoured the group with the meropenem/clavulanate-containing regimens. In addition, synergy with the other anti-TB drugs could have been different for the carbapenems prescribed in this study.

As meropenem/clavulanate appears to be more active against $M$. tuberculosis than imipenem/clavulanate, the former should be favoured in the treatment of MDR-TB. Should meropenem/clavulanate be unavailable, higher doses of imipenem/clavulanate as per Pseudomonas infection could probably be administered (i.e. $1 \mathrm{~g}$ four times daily) given that the drug is well tolerated.

Among the strengths of the study we mention the large size of the imipenem/clavulanate and meropenem/ clavulanate cohorts, and the detailed information collected from the participating centres in Europe and Latin America. The drug resistance patterns, number of previous anti-treatment cycles as well as most of the demographic, epidemiological and clinical variables did not differ significantly in the meropenem/ clavulanate and imipenem/clavulanate cohorts.

However, the observational and retrospective design of the study determines several interpretational limitations. In particular, we mention the missing pre-study definition of a sample size and the possibility to randomise and ensure blindness, which could increase the probability of a selection bias. Furthermore, the latter methodological issue is clearly highlighted by the higher prevalence of resistance to any fluoroquinolone in the imipenem/clavulanate cohort, as well as a higher prevalence of resistance to two
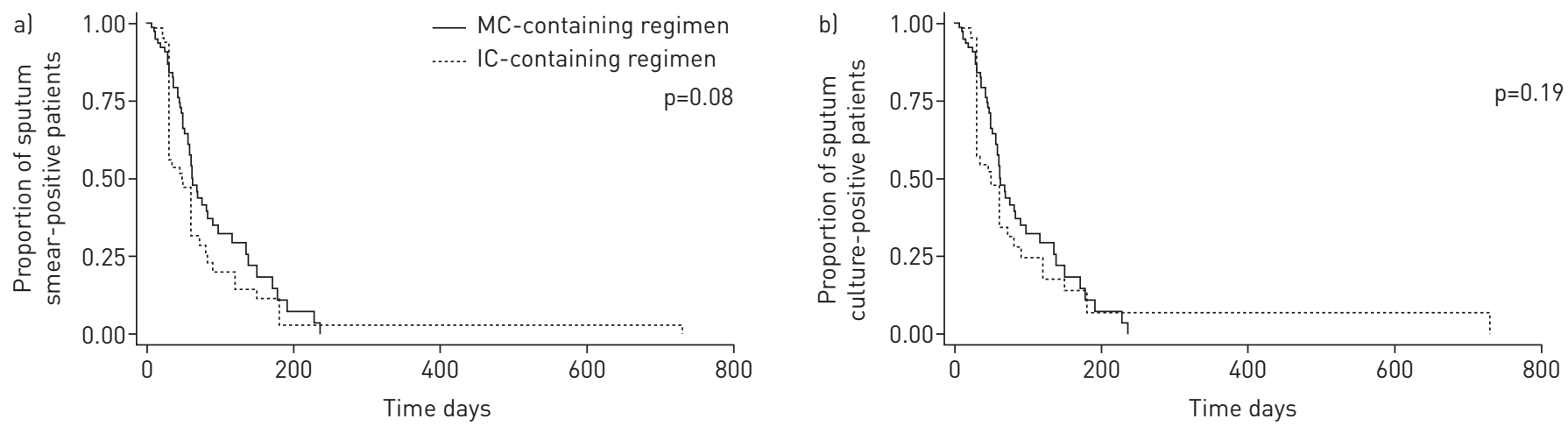

FIGURE 1 a) Sputum smear and b) culture conversion in multidrug- and extensively drug-resistant tuberculosis patients treated with meropenem/ clavulanate (MC)-versus imipenem/clavulanate (IC)-containing regimens. 
out of three second-line anti-TB injectables (kanamycin and capreomycin). Furthermore, exposure to previous anti-TB treatment and the proportion of previous treatment failures was higher in the imipenem/ clavulanate cohort.

As meropenem/clavulanate seems to perform better, but imipenem/clavulanate is cheaper and more widely available, economic analyses will be important to finally assess the future role of these compounds. However, the new information provided by this observational study allows clinicians managing difficult-to-treat $\mathrm{TB}$ cases to know how to use carbapenems and other repurposed drugs in case the minimum number of active drugs necessary to design an effective regimen is lacking [3, 4, 9, 44-47].

In this context, other second-line drugs such as fluoroquinolones or clofazimine might contribute to cardiologic or other adverse events [48]. Unlike all of the above, the repurposed carbapenems are frequently well tolerated and offer few drug-drug interactions, and should the need arise can replace other TB drugs based on their advantageous safety and tolerability profile. The authors believe that the benefits of $\beta$-lactam use for the treatment of TB outweighs the risks of further antimicrobial resistance.

Although new compounds will hopefully appear soon to support the move towards TB elimination [49], the carbapenems are confirmed to have a particular role. The authors would recommend the use of meropenem/clavulanate in the intensive phase of treatment, particularly to manage the most severe cases until bacteriological conversion has been achieved. If active drugs are lacking to design an effective regimen in the continuation phase, ertapenem/clavulanate or faropenem/clavulanate could potentially be considered in the continuation phase. Given the widespread use of amoxicillin/clavulanic acid in the community, DST for meropenem/clavulanate is recommended before using the drug in a regimen.

\section{References}

World Health Organization. Global Tuberculosis Report 2015. Geneva, World Health Organization, 2015.

2 Skrahina A, Hurevich H, Zalutskaya A, et al. Alarming levels of drug-resistant tuberculosis in Belarus: results of a survey in Minsk. Eur Respir J 2012; 39: 1425-1431.

3 Migliori GB, Sotgiu G, Gandhi NR, et al. Drug resistance beyond extensively drug resistant tuberculosis: individual patient data meta-analysis. Eur Respir J 2013; 42: 169-179.

4 Falzon D, Gandhi N, Migliori GB, et al. Resistance to fluoroquinolones and second-line injectable drugs: impact on multidrug-resistant TB outcomes. Eur Respir J 2013; 42: 156-168.

5 Diel R, Rutz S, Castell S, et al. Tuberculosis: cost of illness in Germany. Eur Respir J 2012; 40: 143-115.

6 Diel R, Vandeputte J, de Vries G, et al. Costs of tuberculosis disease in the European Union: a systematic analysis and cost calculation. Eur Respir J 2014; 43: 554-565.

7 Falzon D, Jaramillo E, Schünemann $\mathrm{HJ}$, et al. WHO guidelines for the programmatic management of drug-resistant tuberculosis: 2011 update. Eur Respir J 2011; 38: 516-528.

8 Migliori GB, De Iaco G, Besozzi G, et al. First tuberculosis cases in Italy resistant to all tested drugs. Euro Surveill 2007; 12: E070517.1.

9 Caminero JA, Scardigli A. Classification of antituberculosis drugs: a new proposal based on the most recent evidence. Eur Respir J 2015; 46: 887-893.

10 Sotgiu G, Pontali E, Migliori GB. Linezolid to treat MDR-/XDR-tuberculosis: available evidence and future scenarios. Eur Respir J 2015; 45: 25-29.

11 Pontali E, Sotgiu G, D'Ambrosio L, et al. Bedaquiline and MDR-TB: a systematic and critical analysis of the evidence. Eur Respir J 2016; 47: 394-402.

12 Esposito S, D'Ambrosio L, Tadolini M, et al. ERS/WHO Tuberculosis Consilium assistance with extensively drug-resistant tuberculosis management in a child: case study of compassionate delamanid use. Eur Respir J 2014; 44: 811-815.

13 Skripconoka V, Danilovits M, Pehme L, et al. Delamanid improves outcomes and reduces mortality in multidrug-resistant tuberculosis. Eur Respir J 2013; 41: 1393-1400.

14 Gler MT, Skripconoka V, Sanchez-Garavito E, et al. Delamanid for multidrug-resistant pulmonary tuberculosis. N Engl J Med 2012; 366: 2151-2160.

15 Pym AS, Diacon AH, Tang S-J, et al. Bedaquiline in the treatment of multi- and extensively drug-resistant tuberculosis. Eur Respir J 2016; 47: 564-574.

16 Diacon AH, Pym A, Grobusch M, et al. The diarylquinoline TMC207 for multidrug-resistant tuberculosis. $N$ Engl J Med 2009; 360: 2397-2405.

17 Diacon AH, Pym A, Grobusch MP, et al. Multidrug-resistant tuberculosis and culture conversion with bedaquiline. N Engl J Med 2014; 371: 723-732.

18 Guglielmetti L, Le Dû D, Jachym M, et al. Compassionate use of bedaquiline for the treatment of multidrug-resistant and extensively drug-resistant tuberculosis: interim analysis of a French cohort. Clin Infect Dis 2015; 60: 188-194.

19 Ndjeka N, Conradie F, Schnippel K, et al. Treatment of drug-resistant tuberculosis with bedaquiline in a high HIV prevalence setting: an interim cohort analysis. Int J Tuberc Lung Dis 2015; 19: 979-985.

20 Tiberi S, De Lorenzo S, Centis R, et al. Bedaquiline in MDR/XDR-TB cases: first experience on compassionate use. Eur Respir J 2014; 43: 289-292.

21 Tiberi S, D'Ambrosio L, De Lorenzo S, et al. Tuberculosis elimination, patients' lives and rational use of new drugs: revisited. Eur Respir J 2016; 47: 664-667.

22 Migliori GB, Eker B, Richardson MD, et al. A retrospective TBNET assessment of linezolid safety, tolerability and efficacy in MDR-TB. Eur Respir J 2009; 34: 387-393.

23 Villar M, Sotgiu G, D'Ambrosio L, et al. Linezolid safety, tolerability and efficacy to treat multidrug- and extensively drug-resistant tuberculosis. Eur Respir J 2011; 38: 730-733. 
$$
35
$$

De Lorenzo S, Centis R, D’Ambrosio L, et al. On linezolid efficacy and tolerability. Eur Respir J 2012; 39: 770-772. Sotgiu G, Centis R, D’Ambrosio L, et al. Efficacy, safety and tolerability of linezolid containing regimens in treating MDR-TB and XDR-TB: systematic review and meta-analysis. Eur Respir J 2012; 40: 1430-1442.

Sotgiu G, Centis R, D’Ambrosio L, et al. Linezolid to treat extensively drug-resistant TB: retrospective data are confirmed by experimental evidence. Eur Respir J 2013; 42: 288-290.

Lee M, Lee J, Carroll MW, et al. Linezolid for treatment of chronic extensively drug-resistant tuberculosis. $N$ Engl J Med 2012; 367: 1508-1518.

Sotgiu G, Centis R, D'Ambrosio L, et al. Low minimal inhibitory concentrations of linezolid against multidrug-resistant tuberculosis strains. Eur Respir J 2015; 45: 287-289.

De Lorenzo S, Alffenaar JW, Sotgiu G, et al. Efficacy and safety of meropenem-clavulanate added to linezolid-containing regimens in the treatment of MDR-/XDR-TB. Eur Respir J 2013; 41: 1386-1392.

Payen MC, De Wit S, Martin C, et al. Clinical use of meropenem-clavulanate combination for extensively drug-resistant tuberculosis. Int J Tuberc Lund Dis 2012; 16: 558-560.

1 Tiberi S, D’Ambrosio L, De Lorenzo S, et al. Ertapenem in the treatment of MDR-TB: first clinical experience. Eur Respir J 2016; 47: 333-336.

van Rijn SP, van Altena R, Akkerman OW, et al. Pharmacokinetics evaluation of ertapenem in patients with treatment of multidrug-resistant tuberculosis. Eur Respir J 2016; 47: 1229-1234.

Chambers HF, Turner J, Schecter GF, et al. Imipenem for treatment of tuberculosis in mice and humans. Antimicrob Agents Chemother 2005; 49: 2816-2282.

4 Arbex MA, Ribeiro de Siqueira H, D'Ambrosio L, et al. The challenge of managing the extensively drug-resistant tuberculosis at a referral hospital in the state of São Paulo, Brazil: a report of three cases. J Bras Pneumol 2015; 41: 554-559.

Yew WW, Wong CF, Lee J, et al. Do beta-lactam-beta-lactmase inhibitor combinations have a place in the treatment of multidrug-resistant pulmonary tuberculosis? Tubercle Lung Dis 1995; 78: 90-92.

Hugonnet JE, Blanchard JS. Irreversible inhibition of Mycobacterium tuberculosis beta-lactamase by clavulanate. Biochemistry 2007; 46: 11198-12004.

Hugonnet JE, Tremblay LW, Boshoff HI, et al. Meropenem-clavulanate is effective against extensively drug-resistant Mycobacterium tuberculosis. Science 2009; 323: 1215-1218.

38 Payen M, Martin C, Antoine-Moussiaux T, et al. Four cases of XDR-TB treated with meropenem-clavulanate. 50th Interscience Conference on Antimicrobial Agents and Chemotherapy (ICAAC), 2010. Abstract L1-517. www.abstractsonline.com/Plan/ViewAbstract.aspx?sKey=7189a738-03a3-41e0-8ee5-cf36269b5766\&cKey=aceae86cc8ae-4d8e-968e-d2dd95abed53\&mKey=\%7b93AEED6A-54D4-4EF6-99BD-A9B3CE9FACD9\%7d Date last accessed: April 1, 2016

39 Gonzalo X, Drobniewski F. Is there a place for $\beta$-lactams in the treatment of multidrug-resistant/extensively drug-resistant tuberculosis? Synergy between meropenem and amoxicillin/clavulanate. J Antimicrob Chemother 2013; 68: 366-369.

40 Veziris N, Truffot C, Mainardi J-L, et al. Activity of carbapenems combined with clavulanate against murine tuberculosis. Antimicrob Agents Chemother 2011; 55: 2597-2600.

41 Palmero D, González Montaner P, Cufré M, et al. Primera serie de pacientes con TB-XDR y pre-XDR tratados con esquemas que incluyeron meropenem-clavulanato en Argentina. [First series of patients with XDR and Pre-XDR TB treated with regimens that included meropenen-clavulanate in Argentina.] Arch Bronconeumol 2015; 51: e49-e52.

42 Tiberi S, Payen M-C, Sotgiu G, et al. Effectiveness and safety of meropenem/clavulanate-containing regimens in the treatment of MDR- and XDR-TB. Eur Respir J 2016; 47: 1235-1243.

43 Cordillot M, Dubée V, Triboulet S, et al. In vitro cross-linking of Mycobacterium tuberculosis peptidoglycan by L, D-transpeptidases and inactivation of these enzymes by carbapenems. Antimicrob Agents Chemother 2013; 57: 5940-5945.

44 Winters N, Butler-Laporte G, Menzies D. Efficacy and safety of World Health Organization group 5 drugs for multidrug-resistant tuberculosis treatment. Eur Respir J 2015; 46: 1461-1470.

45 Alsaad N, Wilffert B, van Altena R, et al. Potential antimicrobial agents for the treatment of multidrug-resistant tuberculosis. Eur Respir J 2014; 43: 884-897.

46 Alsaad N, van Altena R, Pranger AD, et al. Evaluation of co-trimoxazole in the treatment of multidrug-resistant tuberculosis. Eur Respir J 2013; 42: 504-512.

47 Krieger D, Vesenbeckh S, Schönfeld N, et al. Mefloquine as a potential drug against multidrug-resistant tuberculosis. Eur Respir J 2015; 46: 1503-1505.

48 Matteelli A, D’Ambrosio L, Centis R, et al. Compassionate and optimum use of new tuberculosis drugs. Lancet Infect Dis 15: 1131-1132.

49 Lonnroth K, Migliori GB, Abubakar I, et al. Towards tuberculosis elimination: an action framework for low-incidence countries. Eur Respir J 2015; 45: 928-952. 